\title{
Approximating Filtered
}

\section{Scale-Variant Signals}

\author{
Alan C. Bovik, Fellow, IEEE, and Raghu G. Raj, Member, IEEE
}

\begin{abstract}
We develop theorems that place limits on the point wise approximation of the responses of filters, both linear shift-invariant (LSI) and linear shift-variant (LSV), to input signals and images that are LSV in the following sense: they can be expressed as the outputs of systems with LSV impulse responses, where the shift-variance is with respect to the filter scale of a single prototype filter. The approximations take the form of LSI approximations to the responses. We develop tight bounds on the approximation errors expressed in terms of filter durations and derivative (Sobolev) norms. Finally, we find application of the developed theory to defoveation of images, deblurring of shift-variant blurs, and shift-variant edge detection.
\end{abstract}

Manuscript received ; revised

A.C. Bovik is with the Laboratory for Image and Video Engineering (LIVE), Department of Electrical and Computer Engineering, The University of Texas at Austin, Austin, TX 78712-1084 USA.

R. Raj is with Applied Research Laboratories, The University of Texas at Austin, Austin, TX 78713-8029 USA

EDICS Category 2-LFLT: Linear Filtering and Enhancement 


\section{INTRODUCTION}

We consider the linear processing of $n$-dimensional signals of the form:

$$
z_{\sigma(\boldsymbol{x})}(\boldsymbol{x})=\frac{1}{[\sigma(\boldsymbol{x})]^{n}} \int_{\boldsymbol{R}^{n}} g[\boldsymbol{a} / \sigma(\boldsymbol{x})] f(\boldsymbol{x}-\boldsymbol{a}) d \boldsymbol{a}
$$

where $\boldsymbol{R}^{n}$ are the $n$-dimensional reals, $\boldsymbol{x}=\left(x_{1}, \ldots, x_{n}\right), f: \boldsymbol{R}^{n} \rightarrow \boldsymbol{R}$ is a continuously-differentiable signal being filtered by the linear filter kernel $g: \boldsymbol{R}^{n} \rightarrow \boldsymbol{R}$, and $\sigma(x): \boldsymbol{R}^{n} \rightarrow \boldsymbol{R}^{+}$is a non-negative, shift-variant scale function. Later, we will also consider discrete-domain $n$-dimensional signals having form analogous to (1) with appropriate substitutions made in the definition of the signals and functions. Clearly, (1) may be regarded as a linear shift-variant filtering of the signal $f$ by the kernel $g$, where the shift-variance is a result of allowing the scale function $\sigma$ to vary with $\boldsymbol{x}$. We therefore refer to (1) as a scale-variant filtering or scale-variant convolution of the signal $f$. We make the notation $g \otimes f$ to denote such a scale-variant convolution, to be distinguished from the usual shift-variant convolution notation $g * f$.

In the sequel it will also be understood that a scale-variant signal refers to a signal that can be written as (1) (or in the corresponding discrete form given in Section III). If $\sigma(\boldsymbol{x})=\sigma=$ constant, then (1) takes the form of the familiar linear shift-invariant convolution

$$
z_{\sigma}(\boldsymbol{x})=\frac{1}{\sigma^{n}} \int_{\boldsymbol{R}^{n}} g(\boldsymbol{a} / \sigma) f(\boldsymbol{x}-\boldsymbol{a}) d \boldsymbol{a} .
$$

Scale-variant signals of the form (1) appear in numerous applications, such as image and video foveation (where images are intentionally non-uniformly blurred) as a method of perceptual compression [1]-[4]; and in modeling undesirable degradations in signals that are blurred non-uniformly, e.g. by coma [5], [6]. Prior work on scale-variant signal processing has consisted mainly in applications to modeling biological vision systems [15]-[17] including fast 
implementation of foveation filtering [18-19]. Scale-variant filtering has also been touched upon in the context of steerable filters [20-21], and log-polar representation of images [22]. Equations (1) and (2) also bear strong resemblance to the continuous wavelet transform [7], [8] if interpreted as a function of scale.

Here we are concerned with linear filtering of signals modeled as the responses of scalevariant linear systems, viz., can be written in the form (1). We will develop theorems that place limits on the approximation of the responses of filters, both linear shift-invariant (LSI) and linear shift-variant (LSV), to input signals and images that are LSV in the sense expressed by (1): that they can be expressed as the outputs of systems with LSV impulse responses, where the shiftvariance is with respect to the filter scale of a single prototype filter. The approximations take the form of LSI approximations to the responses. We develop theorems that express tight bounds on the approximation errors, expressed in terms of filter durations and derivative (Sobolev) norms.

The paper is organized as follows. Section II develops the basic model of filtering scalevariant signals of the form (1) in continuous coordinates. Approximations are given for the outputs of linear systems, both LSI and LSV, to inputs modeled as (1). Tight bounds are developed for the approximation errors. Interestingly, some of these bounds depend on the dimensionality, $n$, of the signal. In Section II, analogous approximations and bounds are discovered for signals defined on discrete coordinates. Section IV finds application of the developed theory to several problems of interest, including defoveation of images, deblurring of shift-variant blurs, and shift-variant edge detection. The paper concludes in Section V.

\section{QUASI-INVARIANT FILTERING OF SCALE-VARIANT SIGNALS}


In this section we develop the continuous-domain notation and theory of approximating linearly filtered scale-variant signals. In the first part, we state the relevant theorems. Proofs are assigned to the Appendix. In the second part, we give analytic examples of the utility of the theoretical results. Numerical results using real signals are given in a later section.

\section{A. Approximations and Theorems}

We consider linear filtering of signals modeled as the responses of scale-variant linear systems, viz., can be written in the form (1). Specifically we study functions of the form

$$
q_{\sigma(x)}(\boldsymbol{x})=\int_{\boldsymbol{R}^{n}} h(\boldsymbol{b}) \cdot \frac{1}{[\sigma(\boldsymbol{x}-\boldsymbol{b})]^{n}} \int_{\boldsymbol{R}^{n}} g[\boldsymbol{a} / \sigma(\boldsymbol{x}-\boldsymbol{b})] f(\boldsymbol{x}-\boldsymbol{b}-\boldsymbol{a}) d \boldsymbol{a} d \boldsymbol{b}
$$

which is the linear filtering (either LSI or LSV) of the scale-variant signal (1) by the kernel $h$.

We shall also be interested in the filtered function

$$
\begin{aligned}
\hat{q} \sigma\left(x_{0}\right)(\boldsymbol{x}) & =h(\boldsymbol{x}) * z_{\sigma\left(x_{0}\right)}(\boldsymbol{x})=h(\boldsymbol{x}) * z_{\sigma}(\boldsymbol{x}) \mid \sigma=\sigma\left(x_{0}\right) \\
& =\int_{\boldsymbol{R}^{n}} h(\boldsymbol{b}) \cdot \frac{1}{\left[\sigma\left(\boldsymbol{x}_{0}\right)\right]^{n}} \int_{\boldsymbol{R}^{n}} g\left[\boldsymbol{a} / \sigma\left(\boldsymbol{x}_{0}\right)\right] f(\boldsymbol{x}-\boldsymbol{b}-\boldsymbol{a}) d \boldsymbol{a} d \boldsymbol{b} .
\end{aligned}
$$

This is the LSI convolution of the filter $h$ with the function $z_{\sigma\left(x_{0}\right)}(\boldsymbol{x})$, but with the scale function held constant: $\sigma(\boldsymbol{x})=\sigma\left(\boldsymbol{x}_{0}\right)=$ constant; hence $z_{\sigma\left(x_{0}\right)}(\boldsymbol{x})$ is space-invariant and (4) is a true double convolution. As indicated by (2), this is also the LSI convolution of $h$ with (2), where $\sigma=\sigma\left(x_{0}\right)$.

The coordinate $\boldsymbol{x}_{0}$ is the point at which we make approximation to the filtered scalevariant signal (3). In fact, the approximation is (4). Thus define the quasi-invariant approximation of $q_{\sigma(x)}(\boldsymbol{x})$ at the point $\boldsymbol{x}_{0}$ :

$$
\left.\hat{q}_{\sigma\left(x_{0}\right)}(x) \approx q_{\sigma(x)}(x)\right|_{x=x_{0}} .
$$


If the filter $h$ and the scale variant signal $z_{\sigma\left(x_{0}\right)}(\boldsymbol{x})$ are such that the approximation (5) is close (in some sense), then (3) will be referred to as a quasi-invariant filtering of the scale-variant signal.

The following Theorem places a bound on the magnitude of the error

$$
\varepsilon\left(x_{0}\right)=\left.q_{\sigma(x)}(x)\right|_{x=x_{0}}-\left.\hat{q} \sigma\left(x_{0}\right)(x)\right|_{x=x_{0}}
$$

of approximation (5). It is expressed in terms of the filter durations and certain derivative or smoothness norms of the signal being filtered and of the scaling function. We define these first.

The $n$-dimensional vector $\Delta \boldsymbol{g}=\left(\Delta g_{1}, \ldots, \Delta g_{n}\right)^{T}$ has elements

$$
\Delta g_{i}=\int_{\boldsymbol{R}^{n}} v_{i}^{2} g^{2}(\boldsymbol{v}) d \boldsymbol{v} ; i=1, \ldots, n
$$

with identical definition for the $\Delta h_{i} ; i=1, \ldots, n$ of $\Delta \boldsymbol{h}$. The elements $\Delta g_{i}$ and $\Delta h_{i}$ are the energy variances (durations) of $g(\boldsymbol{x})$ and $h(\boldsymbol{x})$ along the direction of the axis $x_{i} ; i=1, \ldots, n$.

The vectors $\delta f=\left(\delta f_{1}, \ldots, \delta f_{n}\right)^{T}$ and $\partial \sigma=\left(\partial \sigma_{1}, \ldots, \partial \sigma_{n}\right)^{T}$ have elements

$$
\begin{aligned}
& \delta f_{i}=\int_{\boldsymbol{R}^{n}}\left|\nabla f_{i}(\boldsymbol{r})\right|^{2} d \boldsymbol{r} ; i=1, \ldots, n \\
& \partial \sigma_{i}=\int_{\boldsymbol{R}^{n}} \frac{\left|\nabla \sigma_{i}(\boldsymbol{b})\right|^{2}}{[\sigma(\boldsymbol{b})]^{n}} d \boldsymbol{b} ; i=1, \ldots, n,
\end{aligned}
$$

where $\nabla f_{i}(\boldsymbol{x})=\partial f(\boldsymbol{x}) / \partial x_{i}$ is the $i$ th element of the gradient vector of $f(\boldsymbol{x})$. The elements $\delta f_{i}$ and $\partial \sigma_{i}, i=1, \ldots, n$ are derivative functionals, or Sobolev norms, which are measures of the smoothness of the functions $f(\boldsymbol{x})$ and $\sigma(\boldsymbol{x})$ along the direction of the axis $x_{i} ; i=1, \ldots, n$. The integrands of the functionals $\partial \sigma_{i}$ are weighted by the reciprocal of the scaling function, and so express a greater sensitivity when $\sigma(\boldsymbol{x})$ is small. Finally, given vectors length- $n$ vectors $\Delta \boldsymbol{g}$ and $\delta f$, we denote the vector inner product by $\Delta g \bullet \delta f$. 
Theorem 1 - When $n \neq 2$, the absolute error $\left|\varepsilon\left(x_{0}\right)\right|$ is bounded from above as

$$
\left|\varepsilon\left(x_{0}\right)\right| \leq\left|\frac{2}{2-n}\right| \sqrt{\Delta g \bullet \delta f} \cdot \sqrt{\Delta h \bullet \partial \sigma}
$$

A number of comments regarding this result are in order. First, the bound is tight. As the filters $g(\boldsymbol{x}), h(\boldsymbol{x})$ are taken arbitrarily narrow, the RHS of (10) vanishes. Likewise, if the variation in the signal $f(\boldsymbol{x})$ is sufficiently small, then (10) becomes arbitrarily small: zero if the signal is constant. Likewise, if $\sigma(\boldsymbol{x})$ is made sufficiently smooth, then the bound vanishes.

Secondly, the weighted functional $\partial \sigma$ is of particular interest. At locations $\boldsymbol{x}_{0}$ where the scaling function $\sigma(\boldsymbol{x})$ becomes small, then the bound can become large unless the scale-variant filter or process is such that $\sigma(\boldsymbol{x})$ changes very slowly near $\boldsymbol{x}_{0}$. For example, in image foveation [1]-[4], $\sigma(x)$ increases away from a presumed point of visual fixation (heavier blurring), but may be small near the point of fixation. Theorem 1 implies that at such points, slow changes in $\sigma(\boldsymbol{x})$ are required in order that the approximation (5) might hold accurately. This might be desirable to be able to, e.g., construct an algorithm for de-foveation (as we shall see).

Thirdly, the dependence on the dimensionality $n$ of the involved signals is interesting. For signals of high dimensionality, it appears that the bound (10) becomes very narrow although the involved products may be larger in practice. For $n=2$, the bound is useless! Hence the result seems of reduced interest for two-dimensional signals and images. However, a Corollary result will be given next that provides a useful bound even for $n=2$. Moreover, the bound (10) can be applied to two-dimensional signals when the involved filters are separable, e.g., gaussian.

First, a few more notations are required. Let

$$
\delta f_{\max }=\max _{i} \delta f_{i, \max }
$$




$$
\delta f_{i, \max }=\sup _{\boldsymbol{R}^{n}}\left|\nabla f_{i}(\boldsymbol{r})\right|
$$

and define the alternate directional duration measures

$$
D g_{i}=\int_{\boldsymbol{R}^{n}} v_{i}^{2}|g(\boldsymbol{v})| d \boldsymbol{v} ; i=1, \ldots, n
$$

which are the usual (non-normalized) function variances, and the (directionless) overall duration

$$
D g=\sum_{i=1}^{n} D g_{i}
$$

Corollary 1 - The absolute error $\left|\varepsilon\left(\boldsymbol{x}_{0}\right)\right|$ is bounded from above as

$$
\left|\varepsilon\left(\boldsymbol{x}_{0}\right)\right| \leq n \sqrt{C_{g} \cdot C_{h}} \delta f_{\max } \delta \sigma_{\max } \sqrt{D g \cdot D h}
$$

where

$$
C_{g}=\int_{\boldsymbol{R}^{n}}|g(\boldsymbol{a})| d \boldsymbol{a}, C_{h}=\int_{\boldsymbol{R}^{n}}|h(\boldsymbol{a})| d \boldsymbol{a}
$$

This second result is also dimension-dependent, but with a different (linear) dependence on the dimension $n$ and finite bound when $n=2$. For large $n$, (15) may prove less useful than (10). The bound is again tight in all variables, becoming arbitrarily small as the filter durations are reduced, or as the filter or scale function are made sufficiently smooth.

Making comparisons between the bounds in Theorem 1 and Corollary 1 is difficult, since the durations and the smoothness measures all have distinct definitions, and each contains four terms that behave independently. Nevertheless, the two results substantiate one another, since both indicate that the quasi-invariant filtering approximation of scale-variant signals will tend to be accurate if the involved filters are of short duration, and if the filtered signal is smooth, and if the change in scale is not too rapid. These observations will be born out later in the numerical simulations (Section IV). 


\section{B. Illustrative Examples}

We now examine a few interesting and illustrative examples. These were selected for their general significance and applicability, as opposed to the numerical simulations given later, which demonstrate specific examples of interest.

\section{$\underline{\text { Signal Differentiation }}$}

In numerous applications it is of interest to differentiate a signal, possibly following a linear (and perhaps scale-variant) filtering. For example, in image processing, directional derivatives, gradients or Laplacian operators highlight sustained intensity changes, or edges [9]. In many other applications, derivative operators highlight sudden changes or signal transients, reveal trends, or when combined with nonlinear operations, demodulate AM-FM signals [10].

Suppose that we are given a scale-variant signal (1) with $n=1$. In the context of what is to follow, this would usually be a signal that has been filtered with a linear low-pass (smoothing) function $g$, such as a gaussian, with a varying scale parameter. Suppose then that the scalevariant signal (1) is passed through a $k$-fold differentiator, with impulse response

$$
h(x)=\delta^{(k)}(x)=\frac{d^{k}}{d x^{k}} \delta(x) .
$$

In this case, the quasi-invariant approximation is

$$
\begin{aligned}
\theta_{\sigma\left(x_{0}\right)}(x) & =\left.\delta^{(k)}(x) * z_{\sigma}(x)\right|_{\sigma=\sigma\left(x_{0}\right)} \\
& =\frac{1}{\left[\sigma\left(x_{0}\right)\right]^{n}} g\left[a / \sigma\left(x_{0}\right)\right]^{*} f^{(k)}(x-a),
\end{aligned}
$$

the convolution of the scale-variant filter with the $k^{\text {th }}$ derivative of $f$. If the variation in $f$ and $\sigma$ are finite, as measured by $\delta f_{\text {max }}$ and $\delta \sigma_{\text {max }}$, then (17) is exact when $k \neq 2$, since (15) is zero: $D h$ 
$=0$. When $k=2$, then $D h=\infty$ ! Hence the bound is not applicable, although the approximation remains exact. In this example, (10) is also not applicable for any $k$, since the square of the generalized function (16) is not properly defined [12].

\section{$\underline{\text { Inverse Filtering }}$}

A basic, yet difficult operation in signal processing is the restoration of a signal that has been degraded by a linear distortion function, e.g., image blurred by defocusing or other undesirable smoothing function [11]. The problem is variously called restoration, deconvolution, or inverse filtering, depending on the details of the formulation. The problem is complicated by frequency-domain zeros in the blur function, noise, and other uncertainties that leave the problem generally ill-posed. Yet even more difficult is the case where the blur function is shiftvariant, viz., the degree or the nature of the blur changes from point to point or moment to moment [5], [6]. This problem has been given only a small amount of attention, especially as compared to the case of shift-invariant linear distortion. However, it is of interest for many applications.

We consider the case of attempting to reverse a linear distortion that is scale-variant in the sense of (1). Our approach is to apply an "inverse filter" that is also scale-variant, referred to as scale-variant inverse filter. We first consider the noise-free case where the scale-variant linear distortion is the only degradation of the signal.

Model a signal distorted by $n$-dimensional scale-variant distortion function $g$ using (1). Also assume, for simplicity, that the distortion is suitably well behaved, in the sense that $g$ possesses no frequency-domain zeroes, although this is a practical impossibility. In the future, modifications of the example solution proposed here could be developed, e.g., pseudo-inverse solutions, etc. Denote the Fourier transform of $g$ by 


$$
G(\Omega)=\mathfrak{J}\{g(\boldsymbol{x})\}=\int_{\boldsymbol{R}^{n}} g(a) \exp \left(-j \boldsymbol{\Omega}^{T} \boldsymbol{a}\right) d \boldsymbol{a}
$$

At each $\boldsymbol{x}_{0}, f(\boldsymbol{x})$ is modified by taking the inner product of $f$ with the scaled filter function

$$
g_{\sigma\left(x_{0}\right)}(\boldsymbol{x})=\frac{1}{\left[\sigma\left(\boldsymbol{x}_{0}\right)\right]^{n}} g\left[\boldsymbol{a} / \sigma\left(\boldsymbol{x}_{0}\right)\right]
$$

which has Fourier transform $G_{\sigma\left(x_{0}\right)}(\Omega)=G\left[\sigma\left(x_{0}\right) \Omega\right]$. Then, define the scale-variant inverse

filter

$$
h_{\sigma\left(x_{0}\right)}(\boldsymbol{x})=\mathfrak{J}^{-1}\left\{1 / G\left[\sigma\left(x_{0}\right) \Omega\right]\right\} .
$$

This idea is conceptually simple; at each coordinate, define the scale-variant inverse filter to be the inverse Fourier transform of the reciprocal of the Fourier transform of the filter kernel $g$ scaled by the scaling function evaluated at the current point of interest, $\boldsymbol{x}_{0}$. The idea is that near $\boldsymbol{x}_{0}$, the signal has been modified sufficiently similarly to LSI filtering with $g_{\sigma\left(x_{0}\right)}(\boldsymbol{x})$ that the restoration will be accurate. We note that if $H_{\sigma\left(x_{0}\right)}(\Omega)=\left[1 / G_{\sigma\left(x_{0}\right)}(\Omega)\right]$ is not square integrable, then $H_{\sigma\left(x_{0}\right)}(\Omega)$ is a power-type signal, with an appropriate interpretation for its inverse Fourier transform expressed in terms of generalized functions.

We would be surprised if this idea for shift-variant inverse filtering has not been considered previously; it may even be quite old. However, we have been unable to find a single reference to such a method. In any case, Theorem 1 and Corollary 1 directly address the validity of the approach. The signal $f$ should not vary too quickly; at points where it does, the bounds will be large and the approximation poor. Likewise, the rate of change of the scale of the LSV degradation (and hence of the restoration filters) should be small. Where it changes quickly, expect a poor approximation. The remaining question address the degradation filter durations, and the restoration filter durations. The questions are linked since one defines the other. 
Theorem 1 addresses this question with some generality. The bound (10) is reduced if $\Delta \boldsymbol{g}_{\sigma\left(x_{0}\right)}, \Delta \boldsymbol{h}_{\sigma\left(x_{0}\right)}$ are both small; however, there are limits on how well this can be accomplished.

Dropping the question of scale for simplicity, consider distortion $g \leftrightarrow G$ and inverse filter $h \leftrightarrow$ $H$. By the Fourier transform frequency differentiation theorem and Parseval's formula:

$$
\begin{aligned}
\Delta g_{i}=\int_{\boldsymbol{R}^{n}} v_{i}^{2} g^{2}(\boldsymbol{v}) d \boldsymbol{v}= & \frac{1}{2 \pi} \int_{\boldsymbol{R}^{n}}\left|\frac{\partial}{\partial \Omega_{i}} G(\Omega)\right|^{2} d \Omega \text { for } i=1, \ldots, n \\
\Delta h_{i}=\int_{\boldsymbol{R}^{n}} v_{i}^{2} h^{2}(\boldsymbol{v}) d \boldsymbol{v} & =\frac{1}{2 \pi} \int_{\boldsymbol{R}^{n}}\left|\frac{\partial}{\partial \Omega_{i}} H(\Omega)\right|^{2} d \boldsymbol{\Omega} \\
& =\frac{1}{2 \pi} \int_{\boldsymbol{R}^{n}}\left|\frac{1}{G(\Omega)}\right|^{4}\left|\frac{\partial}{\partial \Omega_{i}} G(\Omega)\right|^{2} d \boldsymbol{\Omega} \text { for } i=1, \ldots, n .
\end{aligned}
$$

While short-duration linear degradation functions might often be encountered in practice, and so (21) might be small, the problem that arises is expressed well by (22): the duration of $h$ is controlled by the reciprocal of $G$. Low-pass blur functions that completely or nearly eradicate high frequencies will have large durations, hence (10) will grow quite large. This is a new interpretation of the main limitation of inverse filters: excessive and unpredictable amplification of high signal frequencies, especially when noise is present. In this case, it limits the reversibility of scale-variant linear degradations and blurs. A consequence of this is that, in cases where scale-variant blurs are intentionally applied to signals, and are desired to be reversible, then the square of the Fourier transforms of the blur functions should not vanish faster than their derivatives.

If the scale-variant blur is accompanied by additive noise, then it is natural to define a scale-variant minimum mean-squared error (MMSE or Wiener) filter, by applying the appropriate MMSE filter at each point in the signal. 


\section{$\underline{\text { Scale-Variant Random Process }}$}

Suppose that $f$ is a wide-sense stationary (wss) random process $f(\boldsymbol{x})=\tilde{f}(\boldsymbol{x})$ with mean $\mu_{f}$ and autocorrelation function $R_{f}(\xi)=\mathrm{E}[\tilde{f}(\boldsymbol{x}) \tilde{f}(\boldsymbol{x}-\xi)]$. The scale-variant filtering (1) delivers a random signal $\widetilde{z}_{\sigma(\boldsymbol{x})}(\boldsymbol{x})$ that is no longer wss.

The mean function of the filtered process is

$$
\begin{aligned}
\mu_{z}(\boldsymbol{x}) & =E\left[\frac{1}{[\sigma(\boldsymbol{x})]^{n}} \int_{\boldsymbol{R}^{n}} g[\boldsymbol{a} / \sigma(\boldsymbol{x})] \tilde{f}(\boldsymbol{x}-\boldsymbol{a}) d \boldsymbol{a}\right] \\
& =\mu_{f} \mu_{g}
\end{aligned}
$$

where

$$
\mu_{g}=\frac{1}{[\sigma(\boldsymbol{x})]^{n}} \int_{\boldsymbol{R}^{n}} g[\boldsymbol{a} / \sigma(\boldsymbol{x})] d \boldsymbol{a}=\int_{\boldsymbol{R}^{n}} g(\boldsymbol{a}) d \boldsymbol{a}=\text { constant }
$$

Since the filters have constant area over scale, then $\widetilde{z}_{\sigma(x)}(\boldsymbol{x})$ has constant mean function.

The autocorrelation function of the output process $\widetilde{z}_{\sigma(x)}(\boldsymbol{x})$ is

$$
\begin{aligned}
& R_{z}(\boldsymbol{x}, \boldsymbol{\xi})=E\left[\widetilde{z}_{\sigma(\boldsymbol{x}-\xi / 2)}(\boldsymbol{x}) \widetilde{z}_{\sigma(\boldsymbol{x}+\boldsymbol{\xi} / 2)}(\boldsymbol{x})\right] \\
& =\frac{1}{[\sigma(\boldsymbol{x}-\boldsymbol{\xi} / 2) \sigma(\boldsymbol{x}+\xi / 2)]^{n}} \int_{\boldsymbol{R}^{n}} \int_{\boldsymbol{R}^{n}} g[\boldsymbol{a} / \sigma(\boldsymbol{x}-\xi / 2)] g[\boldsymbol{b} / \sigma(\boldsymbol{x}+\xi / 2)] E[\tilde{f}(\boldsymbol{x}-\boldsymbol{\xi} / 2-\boldsymbol{a}) \tilde{f}(\boldsymbol{x}+\boldsymbol{\xi} / 2-\boldsymbol{b})] d \boldsymbol{a} d \boldsymbol{b} \\
& =\frac{1}{[\sigma(\boldsymbol{x}-\boldsymbol{\xi} / 2) \sigma(\boldsymbol{x}+\boldsymbol{\xi} / 2)]^{n}} \int_{\boldsymbol{R}^{n}} g[\boldsymbol{a} / \sigma(\boldsymbol{x}-\boldsymbol{\xi} / 2)] \int_{\boldsymbol{R}^{n}} g[\boldsymbol{b} / \sigma(\boldsymbol{x}+\boldsymbol{\xi} / 2)] R_{f}(\boldsymbol{\xi}-\boldsymbol{b}+\boldsymbol{a}) d \boldsymbol{b} d \boldsymbol{a}
\end{aligned}
$$

the inner integral of which is a scale-variant convolution of the form (1). It is of interest to learn whether a useful approximation to (26) can be developed. The outer integral is not a scale- 
variant convolution; therefore we cannot apply Theorem 1 or its Corollary to develop an approximation to (26). However, in a moment we shall state and prove a Lemma that will serve this purpose.

In fact we propose the approximation

$$
\hat{R}_{z}(\boldsymbol{x}, \boldsymbol{\xi})=\frac{1}{[\sigma(\boldsymbol{x})]^{2 n}}\left\{g\left[\frac{-\xi}{\sigma(\boldsymbol{x})}\right] * g\left[\frac{\xi}{\sigma(\boldsymbol{x})}\right] * R_{f}(\xi)\right\}
$$

to $R_{z}(x, \xi)$ which is expressed in terms of shift-invariant convolutions. The autocorrelation approximation (27) is still a function of position $\boldsymbol{x}$; viz., from point-to-point in the signal, the (approximated) correlation structure changes. From a computational perspective, the correlation approximation must be computed via a convolution at every point, but it has the advantage that it need not be computed as a separate operation for every $\xi$ as well, unlike the true expression (26).

The validity of the approximation (27) is addressed by the following Lemma. We denote

$$
\begin{aligned}
& \rho(\boldsymbol{x}, \boldsymbol{\xi})=R_{z}(\boldsymbol{x}, \boldsymbol{\xi})-\hat{R}_{z}(\boldsymbol{x}, \boldsymbol{\xi}) \\
& C_{g}=\int_{\boldsymbol{R}^{n}}|g(\boldsymbol{a})| d \boldsymbol{a} .
\end{aligned}
$$

Lemma 1 - The absolute error $|\rho(x, \xi)|$ is bounded from above as

$$
|\rho(x, \xi)| \leq n \cdot C_{g} \cdot \delta R_{f, \max } \cdot \delta \sigma_{\max } \cdot D g \cdot \sum_{j=1}^{n}\left|\xi_{j}\right|
$$

This result suggests that the formula (27) is most useful for small correlation distances. Indeed, when $\xi=\mathbf{0}$, the approximation is exact. Thus, the approximation captures the second-order point statistics (variances) exactly. The approximation bound is also tight: the error becomes arbitrarily small when the correlation function $R_{f}$ is sufficiently smooth, when the scaling function $g$ changes slowly enough, and when the filter $g$ is adequately narrow. 
As an example of these concepts, we explore the idea of scale-variant zero-crossing rates. If $\tilde{f}(\boldsymbol{x})$ is gaussian, then the output process is gaussian as well. In the case of a onedimensional signal, so that $\widetilde{f}(\boldsymbol{x})=\widetilde{f}(x)$ and $R_{f}(\xi)=R_{f}(\xi)$, the input process has a zero-crossing rate expressed by Rice's famous formula [13]:

$$
\lambda_{0}=\frac{1}{\pi} \sqrt{\frac{R_{f}^{\prime \prime}(0)}{R_{f}(0)}} .
$$

Here we postulate an expression for the shift-variant zero-crossing rate for the case of a onedimensional scale-variant process $\widetilde{z}_{\sigma(x)}(x)$ with approximate autocorrelation function (27). The approximate zero-crossing rate at each $x$ is (naturally enough):

$$
\lambda_{0}(x) \approx \frac{1}{\pi} \sqrt{\frac{\hat{R}_{z}^{\prime \prime}(x, 0)}{\hat{R}_{z}(x, 0)}},
$$

where

$$
\hat{R}_{z}^{\prime \prime}(\boldsymbol{x}, \boldsymbol{\xi})=\frac{1}{[\sigma(x)]^{2 n}}\left\{g^{\prime}\left[\frac{-\xi}{\sigma(x)}\right]^{*} g^{\prime}\left[\frac{\xi}{\sigma(x)}\right] * R_{f}(\xi)\right\}
$$

We have found it difficult to develop an error analysis of the approximation (32), so it remains as a postulate. However, in the simulations, we explore the utility of the approximation for a practical application: zero-crossing based edge detection. While the approximation (32) is 1-D, can also be used to approximate zero-crossing rates along appropriate paths (such as image scan lines) in higher dimensional signals.

\section{DISCRETE FORMULATION}


We now develop results for the case of scale-variant discrete-domain signals filtered by linear filters (LSV or LSI). Consider $n$-dimensional discrete-domain signals of the form:

$$
z_{k(\boldsymbol{m})}(\boldsymbol{m})=\sum_{p \in \boldsymbol{Z}^{n}} g[\boldsymbol{p} / k(\boldsymbol{m})] f(\boldsymbol{m}-\boldsymbol{p}) \quad=g \otimes f
$$

where $\boldsymbol{Z}^{n}$ are the $n$-dimensional integers, $\boldsymbol{m}=\left(m_{1}, \ldots, m_{n}\right), f: \boldsymbol{Z}^{n} \rightarrow \boldsymbol{R}$ is a discrete-domain signal filtered by $g: \boldsymbol{Z}^{n} \rightarrow \boldsymbol{R}$, and $k: \boldsymbol{Z}^{n} \rightarrow \boldsymbol{R}^{+}$is a non-negative, shift-variant integer-valued scaling function. Whenever $\boldsymbol{p} / k(\boldsymbol{m}) \notin \boldsymbol{Z}^{n}$, then we take $g(\boldsymbol{p} / k(\boldsymbol{m}))=0$. We also refer to (34) as a scalevariant filtering or scale-variant convolution of $f$. When $k(\boldsymbol{x})=k=$ constant, then (34) becomes

$$
z_{k}(\boldsymbol{m})=\sum_{\boldsymbol{p} \in \boldsymbol{Z}^{n}} g(\boldsymbol{p} / k) f(\boldsymbol{m}-\boldsymbol{p})
$$

We are concerned with filtering signals of the form (34). Thus we study functions of the form

$$
q_{k(\boldsymbol{m})}(\boldsymbol{m})=\sum_{\boldsymbol{p} \in \boldsymbol{Z}^{n}} h(\boldsymbol{p}) \sum_{\boldsymbol{r} \in \boldsymbol{Z}^{n}} g[\boldsymbol{r} / k(\boldsymbol{m}-\boldsymbol{p})] f(\boldsymbol{m}-\boldsymbol{p}-\boldsymbol{r})
$$

which is the linear (LSI or LSV) filtering of (34) by $h$. We further define

$$
\begin{aligned}
\hat{q}_{k\left(\boldsymbol{m}_{0}\right)}(\boldsymbol{m}) & =h(\boldsymbol{m}) * z_{k\left(\boldsymbol{m}_{0}\right)}(\boldsymbol{m})=\left.h(\boldsymbol{m}) * z_{k}(\boldsymbol{m})\right|_{k=k\left(\boldsymbol{m}_{0}\right)} \\
& =\sum_{\boldsymbol{p} \in \boldsymbol{Z}^{n}} h(\boldsymbol{p}) \sum_{\boldsymbol{r} \in \boldsymbol{Z}^{n}} g\left[\boldsymbol{r} / k\left(\boldsymbol{m}_{0}\right)\right] f(\boldsymbol{m}-\boldsymbol{p}-\boldsymbol{r}) .
\end{aligned}
$$

which has the same explanation as (4): it is the LSI convolution of $h$ with $z_{k\left(\boldsymbol{m}_{0}\right)}(\boldsymbol{m})$, but with the scale function held constant: $k(\boldsymbol{x})=k\left(\boldsymbol{x}_{0}\right)=$ constant. The point $\boldsymbol{m}_{0}$ is where we make approximation to (36); the quasi-invariant approximation is (37):

$$
\left.\hat{q}_{k\left(\boldsymbol{m}_{0}\right)}(\boldsymbol{m}) \approx q_{k(\boldsymbol{m})}(\boldsymbol{m})\right|_{\boldsymbol{m}=\boldsymbol{m}_{0}} .
$$


Again, if filter $h$ and scale variant signal $z_{k\left(\boldsymbol{m}_{0}\right)}(\boldsymbol{m})$ are such that (38) is close, then (36) is a quasiinvariant filtering of the scale-variant signal.

Corollary 2, which follows, bounds the absolute value of the error

$$
\varepsilon\left(\boldsymbol{m}_{0}\right)=\left.q_{k(\boldsymbol{m})}(\boldsymbol{m})\right|_{\boldsymbol{m}=\boldsymbol{m}_{0}}-\left.\hat{q}_{k\left(\boldsymbol{m}_{0}\right)}(\boldsymbol{m})\right|_{\boldsymbol{m}=\boldsymbol{m}_{0}} .
$$

The bound is again expressed in terms of the durations of the involved filters and derivative norms of the signal and scaling function. The discrete directional durations are given

$$
D g_{i}=\sum_{\boldsymbol{r} \in \boldsymbol{Z}^{n}} r_{i}^{2}|g(\boldsymbol{r})| ; i=1, \ldots, n
$$

and the overall duration $D g$ is still given by (14). The overall discrete smoothness functional is

$$
\begin{aligned}
& (\nabla f)_{\max }=\max _{i \in \boldsymbol{Z}, \boldsymbol{s} \in \boldsymbol{Z}^{n}}\left\{\nabla_{i} f(\boldsymbol{s})\right\} \\
& \nabla_{i} f(\boldsymbol{s})=f(\boldsymbol{s})-f\left(\boldsymbol{s}-\Delta_{i}\right)
\end{aligned}
$$

with the vector $\Delta_{i}=(0, \ldots, 1, \ldots, 0)^{T}$ taking nonzero value only in the $i$ th position.

Corollary 2 - The absolute error $\left|\varepsilon\left(\boldsymbol{m}_{0}\right)\right|$ is bounded from above as

$$
\left|\varepsilon\left(\boldsymbol{m}_{0}\right)\right| \leq n(\nabla f)_{\max }(\nabla k)_{\max } \sqrt{D g \cdot D h}
$$

The bound (43) is tight in all terms. For filters $g$ and $h$ taken arbitrarily narrow, the bound vanishes; for signal $f$ and scaling function $k$ taken arbitrarily smooth, it also vanishes.

\section{SIMULATION RESULTS}

In this section we show several examples of the quasi-invariant approximation in simulation. We find practical application to two problems, both suggesting avenues for using the ideas developed here while also serving to exemplify limitations found in schemes based upon such approximations. 


\section{$\underline{\text { Defoveation }}$}

We begin by demonstrating an application of quasi-invariant approximation for defoveation. Foveation can be modeled as a scale-varying filtering system [1]-[4], where the scale of the filter increases away from the point of fixation according to some scaling function.

Figure 1 shows a $512 \times 512$ foveated Lena image with 4 distinct annular regions of filter scales that increase away from the fixation point (presumed to be the image center). The prototype filter used was a unit variance Gaussian filter. Figure 2 shows the defoveated image. The defoveation is performed using the simple scheme described in Section II-B (Inverse Filtering). Figure 3 shows the quasi-invariant approximation. This foveated example was designed to contain sharp discontinuities in the scaling function. It is not representative of a foveation process reflective of the human eye or as appropriate for human consumption. In this case, the defoveation scheme performs poorly, as might be expected from (43).

Figure 4 shows a more representative foveated image using the same prototype filter. Here the foveation is mediated by a gradual change in the filter scale away from fixation. Since the filter scale function varies smoothly, the Sobolev norm of the scale function in (43) is small, hence the quasi-invariant approximation is more accurate, as seen in Figs. 5 and 6.

Now consider the case where there is foveation blur accompanied by additive white Gaussian noise (AWGN). Figure 7 shows a graded foveated image corrupted by AWGN, while Fig. 8 depicts the defoveated image using the scale-variant inverse filters defined above; Fig. 9 shows the defoveated image using MMSE versions of scale-varying inverse filters; clearly, the scale-variant Wiener filtered image (Fig. 9) has much less noise amplification than the scalevariant inverse filtered image (Fig. 8). 


\section{Zero-Crossing Rate Approximation}

As a second type of example, the plausibility of the hypothesis in (32) is demonstrated. Figure 10 depicts plots of the theoretical and the actual zero-crossing rates obtained by applying scale-variant Laplacian-of-Gaussian (LoG) bandpass filters to Gaussian white noise 1-D signals. Here the $\mathrm{ZC}$ rate is plotted against the value of $\sigma$ (expressed in units of sample rate) for the Gaussian filter component of the LoG. As can be seen, the average "theoretical" ZC rate as computed from (32), (33) is in close alignment with the actual ZC rates computed from the scalevariant filtered signals.

As an example of more specific application, note that the ZCs of LoG-filtered images are commonly used for scale-dependent edge detection in images [9]. Figure 11 depicts a scalevariant LoG-filtered image and also the associated ZC map that was computed from it. Although the graded scale-variant LoG was applied to the image tessellated on Cartesian coordinates, the $\mathrm{ZC}$ rates were measured by performing a coordinate transformation into polar coordinates centered at fixation (so that contours of constant radius map to columns). The ZC rate was computed along each row, then the $\mathrm{ZC}$ rates across the rows was averaged. To compute the theoretical ZC rates from (32), (33) the theoretical rate was computed for each $\sigma$ for each row, then these were averaged across rows. Figure 12 shows the plots of theoretical vs. actual ZC rates. It may be noted that the theoretical $\mathrm{ZC}$ rate underestimated the actual $\mathrm{ZC}$ rates in the images; this is likely due to nonstationarities and non-gaussianity in the Lena image.

The implication of these results are that the quasi-invariant approximation may be extended, with care, for extended applications such as ZC rate approximation in scale-variant signals. Such signals can occur, e.g., in foveated edge detection systems.

\section{CONCLUSIONS}


The analysis of the structural responses of systems that depart from the usual assumptions of linearity and/or shift-invariance generally poses significant problems owing to the loss of the principles of superposition and/or frequency-domain equivalence. Analyzing such systems requires either the development of new tools for analysis, which is usually quite difficult, or the use of approximations that relate the systems to other, more easily-analyzed systems. We have taken the second approach here, but we believe that the approximations used are simple enough and sufficiently understandable to find extensive applications. This is particularly likely owing to the increased recognition of the multi-scale (and often scale-variant) structure that is found in signals and images of recorded natural phenomena, such as speech signals, images, and videos.

\section{APPENDIX}

In this Appendix we supply proofs of the main results.

Proof of Theorem 1 - We have that

$$
\begin{aligned}
\left|\varepsilon\left(\boldsymbol{x}_{0}\right)\right|^{2}=\mid & \int_{\boldsymbol{R}^{n}} h(\boldsymbol{b})\left[\int_{\boldsymbol{R}^{n}} f\left(\boldsymbol{x}_{0}-\boldsymbol{b}-\boldsymbol{a}\right) \cdot \frac{1}{\left[\sigma\left(\boldsymbol{x}_{0}\right)\right]^{n}} g\left[\boldsymbol{a} / \sigma\left(\boldsymbol{x}_{0}\right)\right] d \boldsymbol{a}\right. \\
& \left.-\int_{\boldsymbol{R}^{n}} f\left(\boldsymbol{x}_{0}-\boldsymbol{b}-\boldsymbol{a}\right) \cdot \frac{1}{\left[\sigma\left(\boldsymbol{x}_{0}-b\right)\right]^{n}} g\left[\boldsymbol{a} / \sigma\left(\boldsymbol{x}_{0}-b\right)\right] d \boldsymbol{a}\right]\left.d \boldsymbol{b}\right|^{2} .
\end{aligned}
$$

By making appropriate substitutions (A1) becomes:

$$
\left|\varepsilon\left(\boldsymbol{x}_{0}\right)\right|^{2}=\left|\int_{\boldsymbol{R}^{n}} h(\boldsymbol{b}) \int_{\boldsymbol{R}^{n}}\left\{f\left[\boldsymbol{x}_{0}-\boldsymbol{b}-\boldsymbol{v} \sigma\left(\boldsymbol{x}_{0}\right)\right]-f\left[\boldsymbol{x}_{0}-\boldsymbol{b}-\boldsymbol{v} \sigma\left(\boldsymbol{x}_{0}-\boldsymbol{b}\right)\right]\right\} g(\boldsymbol{v}) d \boldsymbol{v} d \boldsymbol{b}\right|^{2}
$$

The term in the inner curly brackets can be evaluated using a first-order Taylor's approximation with explicit remainder [9, p. 203]:

$$
f\left[x_{0}-b-v \sigma\left(x_{0}\right)\right]-f\left[x_{0}-b-v \sigma\left(x_{0}-b\right)\right]
$$




$$
=\int_{0}^{1} \sum_{i=1}^{n} v_{i}\left[\sigma\left(\boldsymbol{x}_{0}-\boldsymbol{b}\right)-\sigma\left(\boldsymbol{x}_{0}\right)\right] \nabla f_{i}\left\{\boldsymbol{x}_{0}-\boldsymbol{b}-\boldsymbol{v} \sigma\left(\boldsymbol{x}_{0}\right)-s \boldsymbol{v}\left[\sigma\left(\boldsymbol{x}_{0}-\boldsymbol{b}\right)-\sigma\left(\boldsymbol{x}_{0}\right)\right]\right\} d s
$$

where $\boldsymbol{v}=\left(v_{1}, \ldots, v_{n}\right)$. With this the squared error (A2) becomes:

$$
\begin{aligned}
& \left|\varepsilon\left(\boldsymbol{x}_{0}\right)\right|^{2}= \\
& \left|\int_{\boldsymbol{R}^{n}} h(\boldsymbol{b})\left[\sigma\left(\boldsymbol{x}_{0}-\boldsymbol{b}\right)-\sigma\left(\boldsymbol{x}_{0}\right)\right] \sum_{i=1}^{n} \int_{0}^{1} \int_{\boldsymbol{R}^{n}} v_{i} g(\boldsymbol{v}) \nabla f_{i}\left\{\boldsymbol{x}_{0}-\boldsymbol{b}-\boldsymbol{v} \sigma\left(\boldsymbol{x}_{0}\right)-s \boldsymbol{v}\left[\sigma\left(\boldsymbol{x}_{0}-\boldsymbol{b}\right)-\sigma\left(\boldsymbol{x}_{0}\right)\right]\right\} d \boldsymbol{v} d s d \boldsymbol{b}\right|^{2}
\end{aligned}
$$

the innermost integral of which is bounded above by the Cauchy-Schwarz inequality:

$$
\begin{aligned}
& \int_{\boldsymbol{R}^{n}} v_{i} g(\boldsymbol{v}) \nabla f_{i}\left\{\boldsymbol{x}_{0}-\boldsymbol{b}-\boldsymbol{v} \sigma\left(\boldsymbol{x}_{0}\right)-s \boldsymbol{v}\left[\sigma\left(\boldsymbol{x}_{0}-\boldsymbol{b}\right)-\sigma\left(\boldsymbol{x}_{0}\right)\right]\right\} d \boldsymbol{v} \\
& \quad \leq \sqrt{\Delta g_{i}} \cdot \sqrt{\int_{\boldsymbol{R}^{n}} \nabla f_{i}^{2}\left\{\boldsymbol{x}_{0}-\boldsymbol{b}-\boldsymbol{v} \sigma\left(\boldsymbol{x}_{0}\right)-s \boldsymbol{v}\left[\sigma\left(\boldsymbol{x}_{0}-\boldsymbol{b}\right)-\sigma\left(\boldsymbol{x}_{0}\right)\right]\right\} d \boldsymbol{v}}
\end{aligned}
$$

where $\Delta g_{i}$ is given in (7). By making the substitutions

$$
\begin{aligned}
& \boldsymbol{r}=\boldsymbol{x}_{0}-\boldsymbol{b}-\boldsymbol{v}\left\{\sigma\left(\boldsymbol{x}_{0}\right)+s\left[\sigma\left(\boldsymbol{x}_{0}-\boldsymbol{b}\right)-\sigma\left(\boldsymbol{x}_{0}\right)\right]\right\} \\
& d \boldsymbol{r}=-\left\{\sigma\left(\boldsymbol{x}_{0}\right)+s\left[\sigma\left(\boldsymbol{x}_{0}-\boldsymbol{b}\right)-\sigma\left(\boldsymbol{x}_{0}\right)\right]\right\} d \boldsymbol{v} .
\end{aligned}
$$

the term inside the radical in (A5) can be re-expressed as

$$
\int_{\boldsymbol{R}^{n}} \nabla f_{i}^{2}\left\{\boldsymbol{x}_{0}-\boldsymbol{b}-\boldsymbol{v} \sigma\left(\boldsymbol{x}_{0}\right)-s \boldsymbol{v}\left[\sigma\left(\boldsymbol{x}_{0}-\boldsymbol{b}\right)-\sigma\left(\boldsymbol{x}_{0}\right)\right]\right\} d \boldsymbol{v}=\frac{1}{\sigma\left(\boldsymbol{x}_{0}\right)+s\left[\sigma\left(\boldsymbol{x}_{0}-\boldsymbol{b}\right)-\sigma\left(\boldsymbol{x}_{0}\right)\right]} \delta f_{i}
$$

where $\delta f_{i}$ is given by (8). Using (A5)-(A9) yields the following bound on the squared error (A2):

$$
\left|\varepsilon\left(\boldsymbol{x}_{0}\right)\right|^{2} \leq \sum_{i=1}^{n} \Delta g_{i} \delta f_{i}\left|\int_{\boldsymbol{R}^{n}} h(\boldsymbol{b})\left[\sigma\left(\boldsymbol{x}_{0}-\boldsymbol{b}\right)-\sigma\left(\boldsymbol{x}_{0}\right)\right] \int_{0}^{1} \frac{d s}{\left\{\sigma\left(\boldsymbol{x}_{0}\right)+s\left[\sigma\left(\boldsymbol{x}_{0}-\boldsymbol{b}\right)-\sigma\left(\boldsymbol{x}_{0}\right)\right]\right\}^{n / 2}} d \boldsymbol{b}\right|^{2}
$$


Making another substitution of variables in the innermost integral of (A10), letting $\Delta \boldsymbol{g}=\left(\Delta g_{1}\right.$ $\left., \ldots, \Delta g_{n}\right)^{T}$ and $\delta f=\left(\delta f_{1}, \ldots, \delta f_{n}\right)^{T}$, then (A10) becomes

$$
\left|c\left(\boldsymbol{x}_{0}\right)\right|^{2} \leq(\Delta \boldsymbol{g} \bullet \delta f)\left|\int_{\boldsymbol{R}^{n}} h(\boldsymbol{b}) \int_{\sigma\left(\boldsymbol{x}_{0}\right)}^{\sigma\left(\boldsymbol{x}_{0}-\boldsymbol{b}\right)} s^{-n / 2} d s d \boldsymbol{b}\right|^{2} .
$$

Further evaluation of (A11) requires noting that the innermost integral yields different definite forms when $n=2$ and $n \neq 2$. When $n \neq 2$,

$$
\int_{\boldsymbol{a}}^{\boldsymbol{b}} s^{-n / 2} d s=\left(\frac{2}{2-n}\right)\left[b^{1-(n / 2)}-a^{1-(n / 2)}\right]
$$

and when $n=2$

$$
\int_{\boldsymbol{a}}^{\boldsymbol{b}} s^{-1} d s=\ln (b / a)
$$

For the case $n \neq 2$, the innermost integral becomes

$$
\begin{gathered}
\int_{\sigma\left(\boldsymbol{x}_{0}\right)}^{\sigma\left(\boldsymbol{x}_{0}-\boldsymbol{b}\right)} s^{-n / 2} d s=\left(\frac{2}{2-n}\right)\left\{\left[\sigma\left(\boldsymbol{x}_{0}\right)\right]^{1-(n / 2)}-\left[\sigma\left(\boldsymbol{x}_{0}-\boldsymbol{b}\right)\right]^{1-(n / 2)}\right\} \\
=\sum_{j=1}^{n} \int_{0}^{1} b_{j} \frac{\nabla \sigma_{j}\left(\boldsymbol{x}_{0}-s \boldsymbol{b}\right)}{\left[\sigma\left(\boldsymbol{x}_{0}-s \boldsymbol{b}\right)\right]^{n / 2}} d s
\end{gathered}
$$

where $\nabla \sigma_{i}(\boldsymbol{x})=\partial \sigma(\boldsymbol{x}) / \partial x_{i}, i=1, \ldots, n$ are the elements of the gradient vector of the scaling function. Equation (A15) follows by application of the closed-form of the first-order Taylor's approximation of the difference within curly brackets in (A14). Hence (A11) becomes $(n \neq 2)$

$$
\left|\varepsilon\left(\boldsymbol{x}_{0}\right)\right|^{2} \leq(\Delta \boldsymbol{g} \bullet \delta f)\left|\int_{0}^{1} \sum_{j=1}^{n} \int_{\boldsymbol{R}^{n}} b_{j} h(\boldsymbol{b}) \frac{\nabla \sigma_{j}\left(\boldsymbol{x}_{0}-s \boldsymbol{b}\right)}{\left[\sigma\left(\boldsymbol{x}_{0}-s \boldsymbol{b}\right)\right]^{n / 2}} d \boldsymbol{b} d s\right|^{2} .
$$


For the case $n=2$, as it turns out, the bound is the same, since

$$
\int_{\sigma\left(\boldsymbol{x}_{0}\right)}^{\sigma\left(\boldsymbol{x}_{0}-\boldsymbol{b}\right)} s^{-1} d s=\ln \left[\sigma\left(\boldsymbol{x}_{0}-\boldsymbol{b}\right) / \sigma\left(\boldsymbol{x}_{0}\right)\right]=\sum_{j=1}^{n} \int_{0}^{1} b_{j} \frac{\nabla \sigma_{j}\left(\boldsymbol{x}_{0}-s \boldsymbol{b}\right)}{\sigma\left(\boldsymbol{x}_{0}-s \boldsymbol{b}\right)} d s
$$

and so it follows that the bound (A11) is given by (A16) for $n=2$ as well. But this can be simplified even further by again applying the Cauchy-Schwarz inequality, this time to the innermost integral of (A16).

$$
\int_{\boldsymbol{R}^{n}} b_{j} h(\boldsymbol{b}) \frac{\nabla \sigma_{j}\left(\boldsymbol{x}_{0}-s \boldsymbol{b}\right)}{\left[\sigma\left(\boldsymbol{x}_{0}-s \boldsymbol{b}\right)\right]^{n / 2}} d \boldsymbol{b} \leq \sqrt{\Delta h_{j}} \cdot \sqrt{\int_{\boldsymbol{R}^{n}} \frac{\left|\nabla \sigma_{j}\left(\boldsymbol{x}_{0}-s \boldsymbol{b}\right)\right|^{2}}{\left[\sigma\left(\boldsymbol{x}_{0}-s \boldsymbol{b}\right)\right]^{n}} d \boldsymbol{b}}
$$

where $\Delta h_{j}$, the directional energy variance of $h(\boldsymbol{x})$, is defined as in (7). Hence the squared error functional (A1) is further bounded as

$$
\left|\varepsilon\left(\boldsymbol{x}_{0}\right)\right|^{2} \leq(\Delta \boldsymbol{g} \bullet \delta f) \sum_{j=1}^{n} \Delta h_{j}\left|\int_{0}^{1} \sqrt{\int_{\boldsymbol{R}^{n}} \frac{\left|\nabla \sigma_{j}\left(\boldsymbol{x}_{0}-s \boldsymbol{b}\right)\right|^{2}}{\left[\sigma\left(\boldsymbol{x}_{0}-s \boldsymbol{b}\right)\right]^{n}}} d \boldsymbol{b} d s\right|^{2}
$$

The innermost integral of (A19) can also be simplified:

$$
\int_{\boldsymbol{R}^{n}} \frac{\left|\nabla \sigma_{j}\left(\boldsymbol{x}_{0}-s \boldsymbol{b}\right)\right|^{2}}{\left[\sigma\left(\boldsymbol{x}_{0}-s \boldsymbol{b}\right)\right]^{n}} d \boldsymbol{b}=\frac{1}{s^{n}} \partial \sigma_{j}
$$

where $\partial \sigma=\left(\partial \sigma_{1}, \ldots, \partial \sigma_{n}\right)^{T}$ is the vector of weighted derivative (Sobolev) norms given by (9). Also denoting $\Delta \boldsymbol{h}=\left(\Delta h_{1}, \ldots, \Delta h_{n}\right)^{T}$, the bound (A19) can be expressed

$$
\left|\varepsilon\left(\boldsymbol{x}_{0}\right)\right|^{2} \leq(\Delta \boldsymbol{g} \bullet \delta f)(\Delta \boldsymbol{h} \bullet \partial \sigma)\left|\int_{0}^{1} s^{-n / 2} d s\right|^{2}
$$


However, $\int_{0}^{1} s^{-n / 2} d s$ evaluates to $2 /(2-n)$ except when $n=2$, in which case the integral does not converge (is infinite). Hence we finally have $(n \neq 2)$

$$
\left|\varepsilon\left(\boldsymbol{x}_{0}\right)\right|^{2} \leq\left(\frac{2}{2-n}\right)^{2}(\Delta \boldsymbol{g} \bullet \delta f)(\Delta \boldsymbol{h} \bullet \partial \sigma)
$$

which, after take the square root of each side, finishes the proof.

Proof of Corollary 1 - We have from (A2) that

$$
\left|\varepsilon\left(\boldsymbol{x}_{0}\right)\right|^{2}=\left|\int_{\boldsymbol{R}^{n}} h(\boldsymbol{b}) \int_{\boldsymbol{R}^{n}} g(\boldsymbol{v})\left\{f\left[\boldsymbol{x}_{0}-\boldsymbol{b}-\boldsymbol{v} \sigma\left(\boldsymbol{x}_{0}\right)\right]-f\left[\boldsymbol{x}_{0}-\boldsymbol{b}-\boldsymbol{v} \sigma\left(\boldsymbol{x}_{0}-\boldsymbol{b}\right)\right]\right\} d \boldsymbol{v} d \boldsymbol{b}\right|^{2}
$$

By the Fundamental Theorem for line integrals we have that

$$
\begin{aligned}
f\left[\boldsymbol{x}_{0}-\boldsymbol{b}-\boldsymbol{v} \sigma\left(\boldsymbol{x}_{0}\right)\right]- & f\left[\boldsymbol{x}_{0}-\boldsymbol{b}-\boldsymbol{v} \sigma\left(\boldsymbol{x}_{0}-\boldsymbol{b}\right)\right] \\
= & \int_{\boldsymbol{x}_{0}-\boldsymbol{b}-\boldsymbol{v} \sigma\left(\boldsymbol{x}_{0}-\boldsymbol{b}\right)}^{\boldsymbol{x}_{0}-\boldsymbol{b}-\boldsymbol{v} \sigma\left(\boldsymbol{x}_{0}\right)} \nabla \boldsymbol{f} \bullet \boldsymbol{d s}=\sum_{i=1}^{n} \int_{-v_{i} \sigma\left(\boldsymbol{x}_{0}-\boldsymbol{b}\right)}^{-v_{i} \sigma\left(\boldsymbol{x}_{0}\right)} \nabla_{i} f(\boldsymbol{x}) d x_{i} .
\end{aligned}
$$

The squared error (A23) can be bounded as

$$
\begin{aligned}
\left|\varepsilon\left(\boldsymbol{x}_{0}\right)\right|^{2} & \leq\left[\sum_{i=1}^{n} \int_{\boldsymbol{R}^{n}}|h(\boldsymbol{b})| \int_{\boldsymbol{R}^{n}}|g(\boldsymbol{v})| \int_{-v_{i} \sigma\left(\boldsymbol{x}_{0}-\boldsymbol{b}\right)} \nabla_{i} f(\boldsymbol{x}) d x_{i} \mid d \boldsymbol{v} d \boldsymbol{b}\right]^{2} \\
& \leq \delta f_{\max }^{2}\left[\sum_{i=1}^{n} \int_{\boldsymbol{R}^{n}}|h(\boldsymbol{b})| \int v_{\boldsymbol{R}^{n}}\left|v_{i}\right| g(\boldsymbol{v})|| \sigma\left(\boldsymbol{x}_{0}-\boldsymbol{b}\right)-\sigma\left(\boldsymbol{x}_{0}\right) \mid d \boldsymbol{v} d \boldsymbol{b}\right]^{2} \\
& =\delta f_{\max }^{2}\left[\sum_{i=1}^{n} \int_{\boldsymbol{R}^{n}}\left|v_{i}\right||g(\boldsymbol{v})| d \boldsymbol{v} \int_{\boldsymbol{R}^{n}}|h(\boldsymbol{b})|\left|\sigma\left(\boldsymbol{x}_{0}-\boldsymbol{b}\right)-\sigma\left(\boldsymbol{x}_{0}\right)\right| d \boldsymbol{b}\right]^{2}
\end{aligned}
$$


where $\delta f_{\max }$ is defined in (11), (12). Defining $\delta \sigma_{\max }$ in this way we can write

$$
\left|\sigma\left(\boldsymbol{x}_{0}-\boldsymbol{b}\right)-\sigma\left(\boldsymbol{x}_{0}\right)\right| \leq \delta \sigma_{\max }\left|\sum_{j=1}^{n} b_{j}\right| \leq \delta \sigma_{\max } \sum_{j=1}^{n}\left|b_{j}\right|
$$

Then, using (A28) and by separating the sums and integrals the squared error (A23) is further bounded as:

$$
\begin{aligned}
\left|\delta\left(\boldsymbol{x}_{0}\right)\right|^{2} & \leq \delta f_{\max }^{2} \delta \sigma_{\max }^{2}\left[\sum_{i=1}^{n} \sum_{j=1}^{n} \int_{\boldsymbol{R}^{n}}\left|v_{i}\right||g(\boldsymbol{v})| d \boldsymbol{v} \int_{\boldsymbol{R}^{n}}\left|b_{i}\right||h(\boldsymbol{b})| d \boldsymbol{b}\right]^{2} \\
& =\delta f_{\max }^{2} \delta \sigma_{\max }^{2}\left[\sum_{i=1}^{n} \int_{\boldsymbol{R}^{n}}\left|v_{i}\right||g(\boldsymbol{v})| d \boldsymbol{v}\right]^{2}\left[\sum_{j=1}^{n} \underset{\boldsymbol{R}^{n}}{\int}\left|b_{i}\right||h(\boldsymbol{b})| d \boldsymbol{b}\right]^{2} .
\end{aligned}
$$

Using this special case of the Cauchy-Schwarz inequality:

$$
\left|\sum_{i=1}^{n} a_{i}\right|^{2} \leq n \sum_{i=1}^{n}\left|a_{i}\right|^{2}
$$

then

$$
\begin{aligned}
\left|\varepsilon\left(\boldsymbol{x}_{0}\right)\right|^{2} & \leq n^{2} \delta f_{\max }^{2} \delta \sigma_{\max }^{2}\left[\sum_{i=1}^{n}\left(\int v_{i}|| g(\boldsymbol{v}) \mid d \boldsymbol{v}\right)^{2}\right]\left[\sum_{j=1}^{n}\left(\int_{\boldsymbol{R}^{n}}\left|b_{i}\right||h(\boldsymbol{b})| d \boldsymbol{b}\right)^{2}\right] \\
& \leq \mathrm{C}_{\mathrm{g}} \mathrm{C}_{\mathrm{h}} n^{2} \delta f_{\max }^{2} \delta \sigma_{\max }^{2} \sum_{i=1}^{n} \int_{\boldsymbol{R}^{n}} v_{i}^{2}|g(\boldsymbol{v})| d \boldsymbol{v} \cdot \sum_{j=1}^{n} \int_{\boldsymbol{R}^{n}} b_{j}^{2}|h(\boldsymbol{b})| d \boldsymbol{b}
\end{aligned}
$$

where the second inequality (A33) follows since for a positive function $r(\boldsymbol{x})$ it is true that

$$
\left[\int_{\boldsymbol{R}^{n}}\left|x_{i}\right| r(\boldsymbol{x}) d \boldsymbol{x}\right]^{2} \leq C_{r} \int_{\boldsymbol{R}^{n}} x_{i}^{2} r(\boldsymbol{x}) d \boldsymbol{x} \quad \text { where } C_{r}=\int_{\boldsymbol{R}^{n}} r(\boldsymbol{x}) d \boldsymbol{x}
$$


Therefore, we finally have

$$
\left|\varepsilon\left(\boldsymbol{x}_{0}\right)\right|^{2} \leq n^{2} C_{g} C_{h} \delta f_{\max }^{2} \delta \sigma_{\max }^{2} D g \cdot D h
$$

which, upon taking square roots, completes the proof.

Proof of Lemma 1 - With some simple substitutions we have that

$|\rho(x, \xi)|^{2}=$

$$
\begin{aligned}
& \left|\int_{\boldsymbol{R}^{n}} g(\boldsymbol{a}) \int_{\boldsymbol{R}^{n}} g(\boldsymbol{b})\left\{R_{f}[\boldsymbol{\xi}-\boldsymbol{b} \sigma(\boldsymbol{x}+\boldsymbol{\xi})+\boldsymbol{a} \sigma(\boldsymbol{x})]-R_{f}[\boldsymbol{\xi}-\boldsymbol{b} \sigma(\boldsymbol{x})+\boldsymbol{a} \sigma(\boldsymbol{x})]\right\} d \boldsymbol{b} d \boldsymbol{a}\right|^{2}(\mathrm{~A}, 6 \\
\leq & {\left[\int_{\boldsymbol{R}^{n}}|g(\boldsymbol{a})| \int_{\boldsymbol{R}^{n}}\left|g(\boldsymbol{b}) \| R_{f}[\xi-\boldsymbol{b} \sigma(\boldsymbol{x}+\boldsymbol{\xi})+\boldsymbol{a} \sigma(\boldsymbol{x})]-R_{f}[\xi-\boldsymbol{b} \sigma(\boldsymbol{x})+\boldsymbol{a} \sigma(\boldsymbol{x})]\right| d \boldsymbol{b} d \boldsymbol{a}\right]^{2} }
\end{aligned}
$$

Reasoning as in (A24), (A25) we have that

$$
\begin{aligned}
|\rho(\boldsymbol{x}, \boldsymbol{\xi})|^{2} & \left.\leq \delta R_{f, \text { max }}^{2}\left[\int_{\boldsymbol{R}^{n}}|g(\boldsymbol{a})| \int_{\boldsymbol{R}^{n}}\left|\sum_{i=1}^{n} b_{i}\right||g(\boldsymbol{b})| \mid \sigma(\boldsymbol{x}+\boldsymbol{\xi})-\sigma(\boldsymbol{x})\right] \mid d \boldsymbol{b} d a\right]^{2} \\
& \leq C_{g}^{2} \delta R_{f, \text { max }}^{2} \delta \sigma_{\max }^{2}\left[\sum_{j=1}^{n}\left|\xi_{j}\right|\right]^{2}\left[\int_{\boldsymbol{R}^{n}}\left|\sum_{i=1}^{n} b_{i}\right||g(\boldsymbol{b})| d \boldsymbol{b}\right]^{2} \\
& \leq n^{2} C_{g}^{2} \delta R_{f, \max }^{2} \delta \sigma_{\max }^{2}\left[\sum_{j=1}^{n}\left|\xi_{j}\right|\right]^{2}\left[\sum_{i=1}^{n} \int_{\boldsymbol{R}^{n}}\left|b_{i}\right|^{2}|g(\boldsymbol{b})| d\right]^{2} \\
& =n^{2} C_{g}^{2} \delta R_{f, \text { max }}^{2} \delta \sigma_{\text {max }}^{2}(D g)^{2}\left[\sum_{j=1}^{n}\left|\xi_{j}\right|\right]^{2}
\end{aligned}
$$

using (A28) and (A31). The proof is finished by taking the square root of both sides of (A41). 
Proof of Corollary 2 - The squared error can be written

$$
\begin{aligned}
\left|g\left(\boldsymbol{m}_{0}\right)\right|^{2} & =\mid \sum_{\boldsymbol{p} \in \boldsymbol{Z}^{n}} h(\boldsymbol{p})\left[\sum_{\boldsymbol{r} \in \boldsymbol{Z}^{n}} f\left(\boldsymbol{m}_{0}-\boldsymbol{p}-\boldsymbol{r}\right) g\left[\boldsymbol{r} / k\left(\boldsymbol{m}_{0}\right)\right]-\sum_{\boldsymbol{r} \in \boldsymbol{Z}^{n}} f\left(\boldsymbol{m}_{0}-\boldsymbol{p}-\boldsymbol{r}\right) g\left[\boldsymbol{r} / k\left(\boldsymbol{m}_{0}-\boldsymbol{p}\right)\right]||^{2}(\mathrm{~A}\right. \\
& =\left|\sum_{\boldsymbol{p} \in \boldsymbol{Z}^{n}} h(\boldsymbol{p})\left[\sum_{\boldsymbol{r} \in \boldsymbol{Z}^{n}} g(\boldsymbol{r}) f\left[\boldsymbol{m}_{0}-\boldsymbol{p}-\boldsymbol{r k}\left(\boldsymbol{m}_{0}\right)\right]-f\left[\boldsymbol{m}_{0}-\boldsymbol{p}-\boldsymbol{r k}\left(\boldsymbol{m}_{0}-\boldsymbol{p}\right)\right]\right]\right|^{2}
\end{aligned}
$$

Reasoning similar to previous proofs, we have:

$$
\left|f\left[\boldsymbol{m}_{0}-\boldsymbol{p}-\boldsymbol{r} k\left(\boldsymbol{m}_{0}\right)\right]-f\left[\boldsymbol{m}_{0}-\boldsymbol{p}-\boldsymbol{r} k\left(\boldsymbol{m}_{0}-\boldsymbol{p}\right)\right]\right|=\left|\sum_{i=1}^{n} \sum_{s_{i}=r_{i} k\left(\boldsymbol{m}_{0}\right)}^{r_{i} k\left(\boldsymbol{m}_{0}-\boldsymbol{p}\right)} \nabla_{i} f(\boldsymbol{s})\right|
$$

so that the squared error (A41) can be bounded

$$
\begin{aligned}
& \left|\varepsilon\left(\boldsymbol{m}_{0}\right)\right|^{2} \leq\left[\sum_{i=1}^{n} \sum_{\boldsymbol{p} \in \boldsymbol{Z}^{n}}|h(\boldsymbol{p})|\left[\sum_{\boldsymbol{r} \in \boldsymbol{Z}^{n}}|g(\boldsymbol{r})|\left|\sum_{s_{i}=r_{i} k\left(\boldsymbol{m}_{0}\right)}^{r_{i} k\left(\boldsymbol{m}_{0}-\boldsymbol{p}\right)} \nabla_{i} f(\boldsymbol{s})\right|\right]\right]^{2} \\
& \leq(\nabla f)_{\max }^{2}\left[\sum_{i=1}^{n} \sum_{\boldsymbol{p} \in \boldsymbol{Z}^{n}}|h(\boldsymbol{p})|\left[\sum_{\boldsymbol{r} \in \boldsymbol{Z}^{n}}\left|r_{i}\right||g(\boldsymbol{r})|\left|k\left(\boldsymbol{m}_{0}-\boldsymbol{p}\right)-k\left(\boldsymbol{m}_{0}\right)\right|\right]\right]^{2} \\
& =(\nabla f)_{\max }^{2}\left[\sum_{i=1}^{n} \sum_{\boldsymbol{r} \in \boldsymbol{Z}^{n}}\left|r_{i}\right||g(\boldsymbol{r})| \sum_{\boldsymbol{p} \in \boldsymbol{Z}^{n}}|h(\boldsymbol{p})|\left|k\left(\boldsymbol{m}_{0}-\boldsymbol{p}\right)-k\left(\boldsymbol{m}_{0}\right)\right|\right]^{2}
\end{aligned}
$$

using (38) and (39). Now

$$
\left|k\left(\boldsymbol{m}_{0}-\boldsymbol{p}\right)-k\left(\boldsymbol{m}_{0}\right)\right| \leq(\nabla k)_{\max } \sum_{j=1}^{n}\left|p_{j}\right|
$$

where $(\nabla k)_{\max }$ is defined as in (38), (39). Hence (A46) is further bounded as 


$$
\begin{aligned}
\left|\varepsilon\left(\boldsymbol{m}_{0}\right)\right|^{2} & \leq(\nabla f)_{\max }^{2}(\nabla k)_{\max }^{2}\left[\sum_{i=1}^{n} \sum_{\boldsymbol{r} \in \boldsymbol{Z}^{n}}\left|r_{i}\right| \mid g(\boldsymbol{r})\right]^{2} \cdot\left[\sum_{j=1}^{n} \sum_{\boldsymbol{p} \in \boldsymbol{Z}^{n}}\left|p_{j}\right||h(\boldsymbol{p})|\right]^{2} \\
& \leq n^{2}(\nabla f)_{\max }^{2}(\nabla k)_{\max }^{2} \sum_{i=1}^{n}\left[\sum_{\boldsymbol{r} \in \boldsymbol{Z}^{n}}\left|r_{i}\right||g(\boldsymbol{r})|\right]^{2} \cdot \sum_{j=1}^{n}\left[\sum_{\boldsymbol{p} \in \boldsymbol{Z}^{n}}\left|p_{j}\right| h(\boldsymbol{p}) \mid\right]^{2} \\
& \leq n^{2}(\nabla f)_{\max }^{2}(\nabla k)_{\max }^{2} \sum_{i=1}^{n} \sum_{\boldsymbol{r} \in \boldsymbol{Z}^{n}} r_{i}^{2}|g(\boldsymbol{r})| \cdot \sum_{j=1}^{n} \sum_{\boldsymbol{p} \in \boldsymbol{Z}^{n}} p_{j}^{2}|h(\boldsymbol{p})| \\
& \leq n^{2}(\nabla f)_{\max }^{2}(\nabla k)_{\max }^{2} D g \cdot D h
\end{aligned}
$$

using (A31) and the discrete version of (A34), (37) and (14). The proof is then finished by taking square roots.

\section{REFERENCES}

[1] W.N. Klarquist and A.C. Bovik, "FOVEA: A foveated, multi-fixation, vergent active stereo system for dynamic three-dimensional scene recovery," IEEE Transactions on Robotics and Automation, vol. 14, no. 5, pp. 755-770, October 1998.

[2] S. Lee, M.S. Pattichis and A.C. Bovik, "Optimal rate control for real-time, low bitrate foveated video coding," IEEE Transactions on Image Processing, vol. 10, no. 7, pp. 977992, July 2001.

[3] S. Lee, M.S. Pattichis and A.C. Bovik, "Foveated video quality assessment," IEEE Transactions on Multimedia, vol. 4, no. 1, pp. 129-132, March 2002.

[4] Z. Wang and A.C. Bovik, "Embedded foveation image coding," IEEE Transactions on Image Processing, vol. 10, no. 10, pp. 1397-1410, October 2001.

[5] G.M. Robbins, "Image restoration for a class of linear spatially variant degradations," Pattern Recognition, vol. 2, pp. 91-103, 1970.

[6] A.A. Sawchuck, "Space-variant image restoration by coordinate transformation," J. Opt. 
Soc. Am., vol. 64, pp. 138-144, Feb. 1974.

[7] A. Grossman and J. Morlet, "Decomposition of Hardy functions into square integrable wavelets of constant shape,” SIAM J. Math. Anal., vol. 15, no. 4, pp. 723-736, July 1984.

[8] S. Mallat, A Wavelet Tour of Signal Processing. Academic Press, 1999.

[9] B.K.P. Horn, Robot Vision. MIT Press, 1985.

[10] A.C. Bovik, P. Maragos and T.F. Quatieri, "AM-FM energy detection and separation in noise using multiband energy operators," IEEE Transactions on Signal Processing, Special Issue on Wavelets and Signal Processing, vol. 41, no. 12, pp. 3245-3265, December 1993.

[11] A.C. Bovik, The Handbook of Image and Video Processing. Academic Press, 2000.

[12] A. Papoulis, The Fourier Integral and its Applications. McGraw-Hill, 1962.

[13] S.O. Rice, "Mathematical analysis of random noise," Bell Syst. Tech. J., vol. 23, pp. 282332, July 1944; and vol. 24, pp. 46-156, Jan. 1945.

[14] R. Etienne-Cummings, J. Van der Spiegel, P. Mueller and M.Z. Zhang, “A foveated silicon retina for two-dimensional tracking," IEEE Trans. Circ. Syst. II, vol. 47, pp. 504-517, June 2000.

[15] M. Bolduc and M. D. Levine. "A review of biologically motivated space-variant data reduction models for robotic vision," Computer Vision and Image Understanding, vol. 69, pp. $170-184,1998$.

[16] C. Braccini, G. Gambardella, and G. Sandini. "A signal theory approach to the space and frequency variant filtering performed by the human visual system," Signal Processing, vol. 3, pp. 231-240, 1981.

[17] P. Perona. "Deformable kernels for early vision," IEEE Trans. on Patt. Anal. and Mach. Intell., vol. 17, no. 5, pp. 488-499, 1995.

[18] W. S. Geisler and J. S. Perry. "A real-time foveated multiresolution system for low- 
bandwidth video communication," in Proc. of the SPIE, vol. 3299, pp. 294-305, San Jose, CA, Jan. 1998.

[19] P. Kortum and W. Geisler. "Implementation of a foveated image coding system for image bandwidth reduction," in Proc. of the SPIE, vol. 2657, pp. 350-360, San Jose, CA, Jan. 1996.

[20] W. T. Freeman and E. H. Adelson. "The design and use of steerable filters," IEEE Trans. On Patt. Anal. and Mach. Intell., vol. 13, no. 9, pp. 891-906, 1991.

[21] E. P. Simoncelli, W. T. Freeman, E. H. Adelson, and D.J. Heeger. "Shiftable multiscale transforms," IEEE Transactions on Information Theory, vol. 38, no. 2, pp. 587-607, 1992.

[22] A. Tabernero, J. Portilla, and R. Navarro. "Duality of a log-polar image representation in the space and the spatial frequency domains," IEEE Trans. on Sign. Proc., vol. 47, pp. 2469-79, Sep. 1999. 


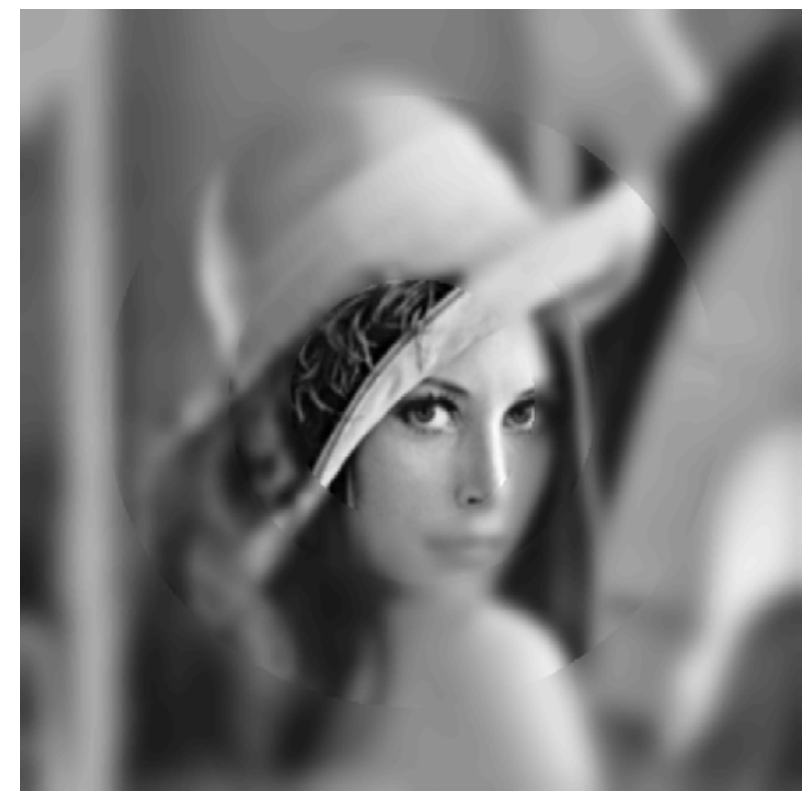

Fig. 1. Foveated image (with 4 annular regions).

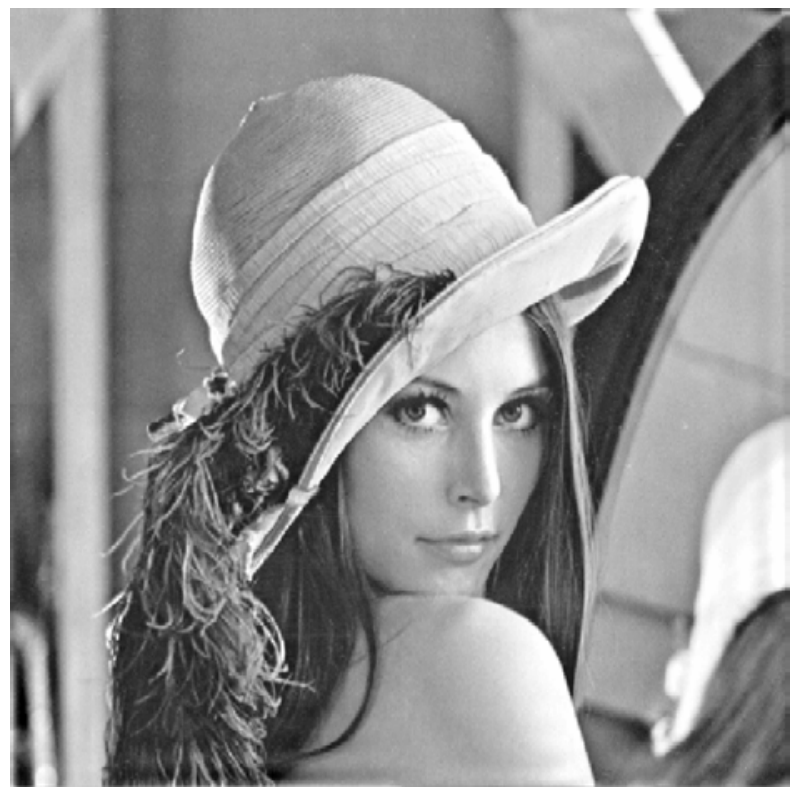

Fig. 3. Quasi-invariant approximation to the defoveated image.

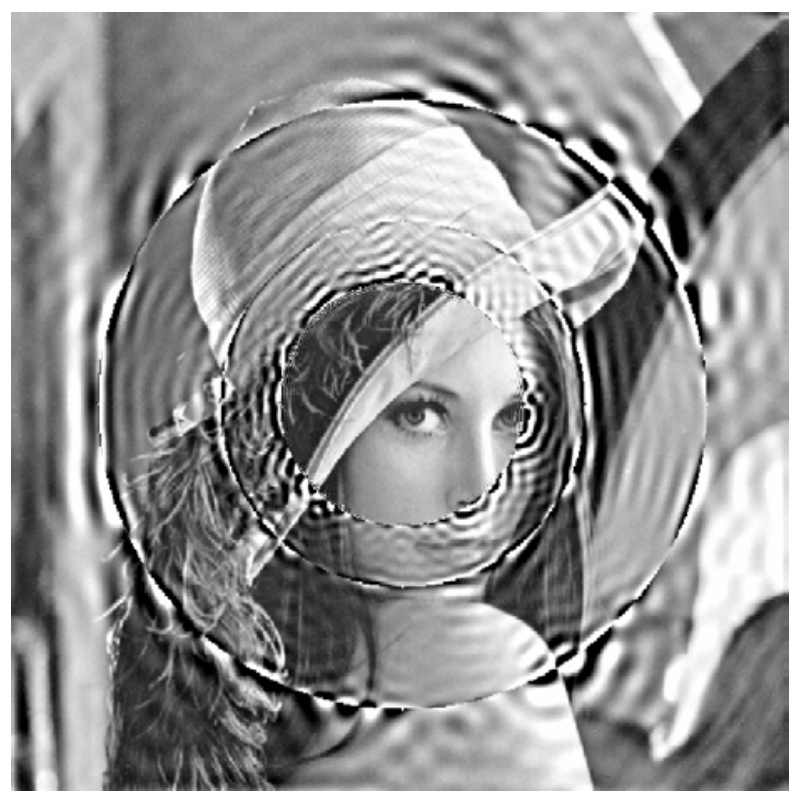

Fig. 2. Defoveated image.

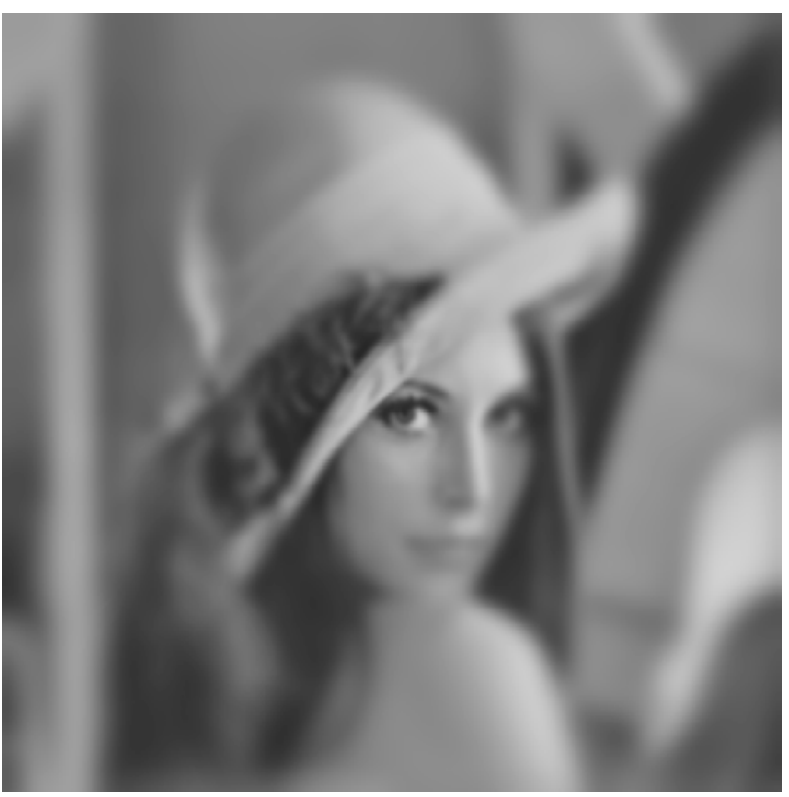

Fig. 4. Graded foveated image. 




Fig. 5. Defoveation of graded foveated image.

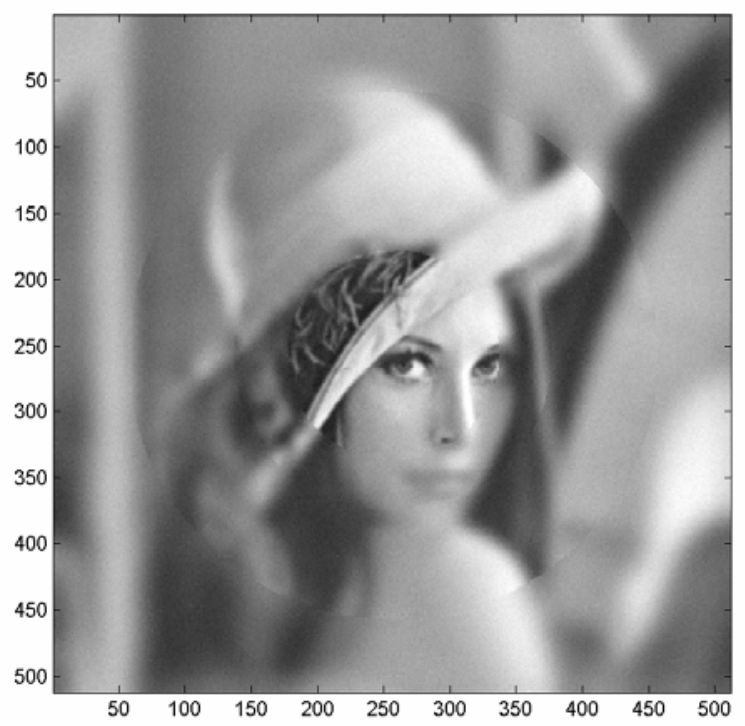

Fig.7. Image foveated over four annular regions corrupted by AWGN (variance $=10.0)$

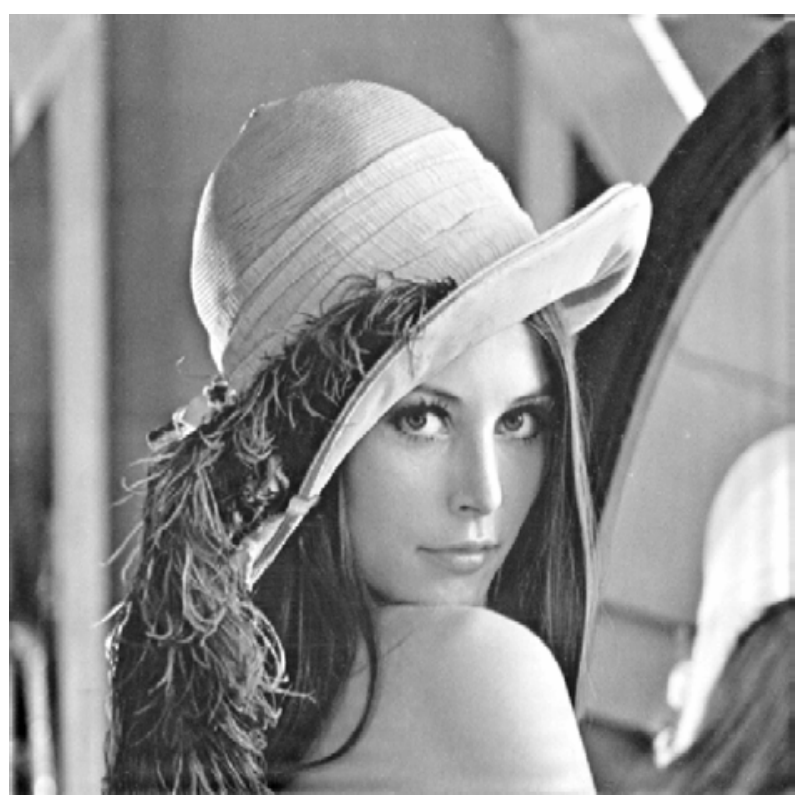

Fig. 6. Quasi-invariant approximation of graded defoveated image.



Fig. 8. Defoveated version of graded, foveated noisy image in Fig. 7. 


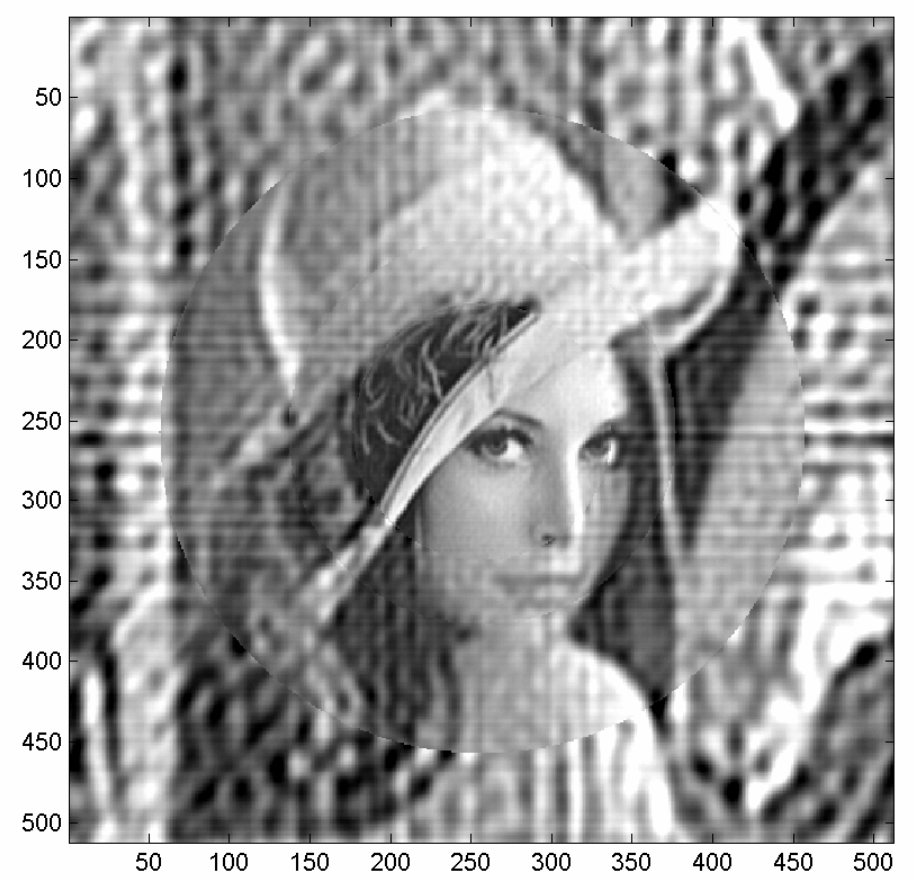

Fig. 9. MMSE (Wiener) defoveated version of graded, foveated noisy image in Fig. 7.

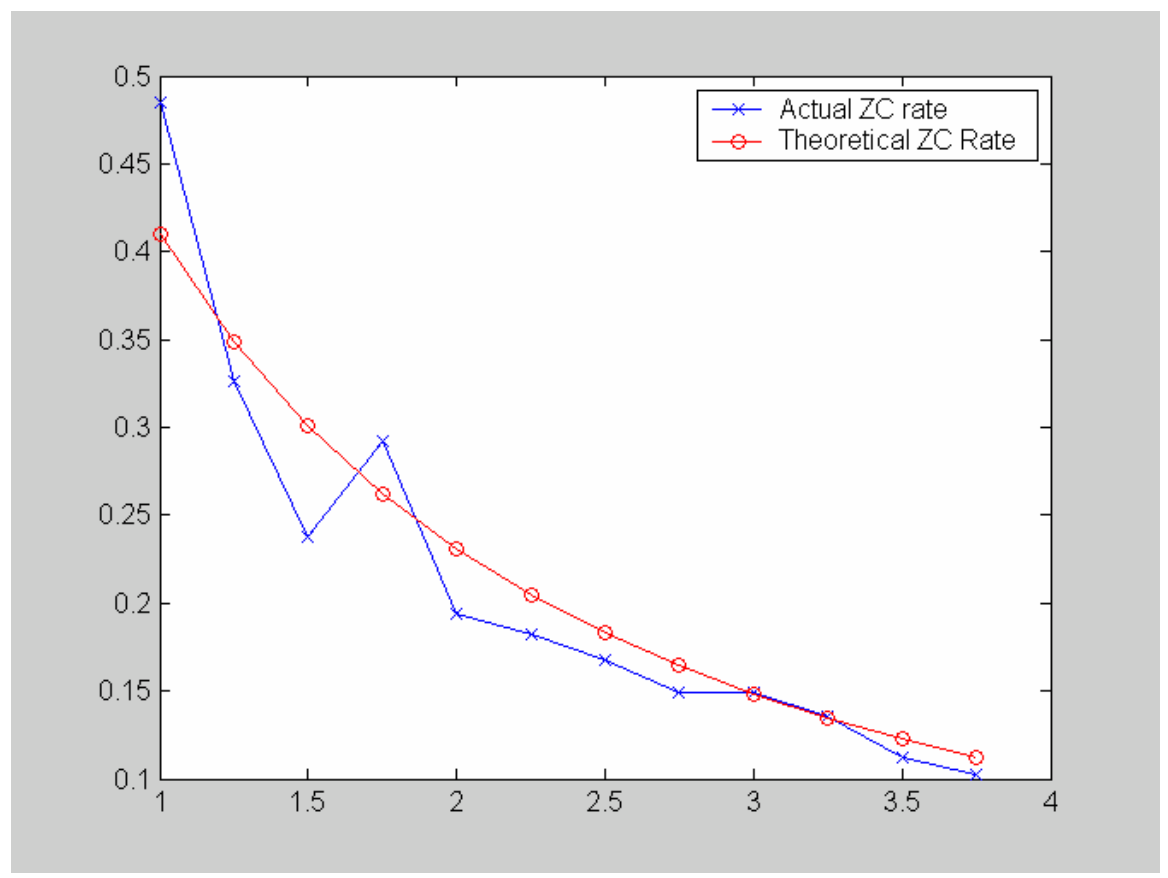

Fig. 10. Plot of theoretical and actual zero-crossing rates averaged over 100 1-D Gaussian noise signals filtered by scale-variant linear Gaussian filters. 

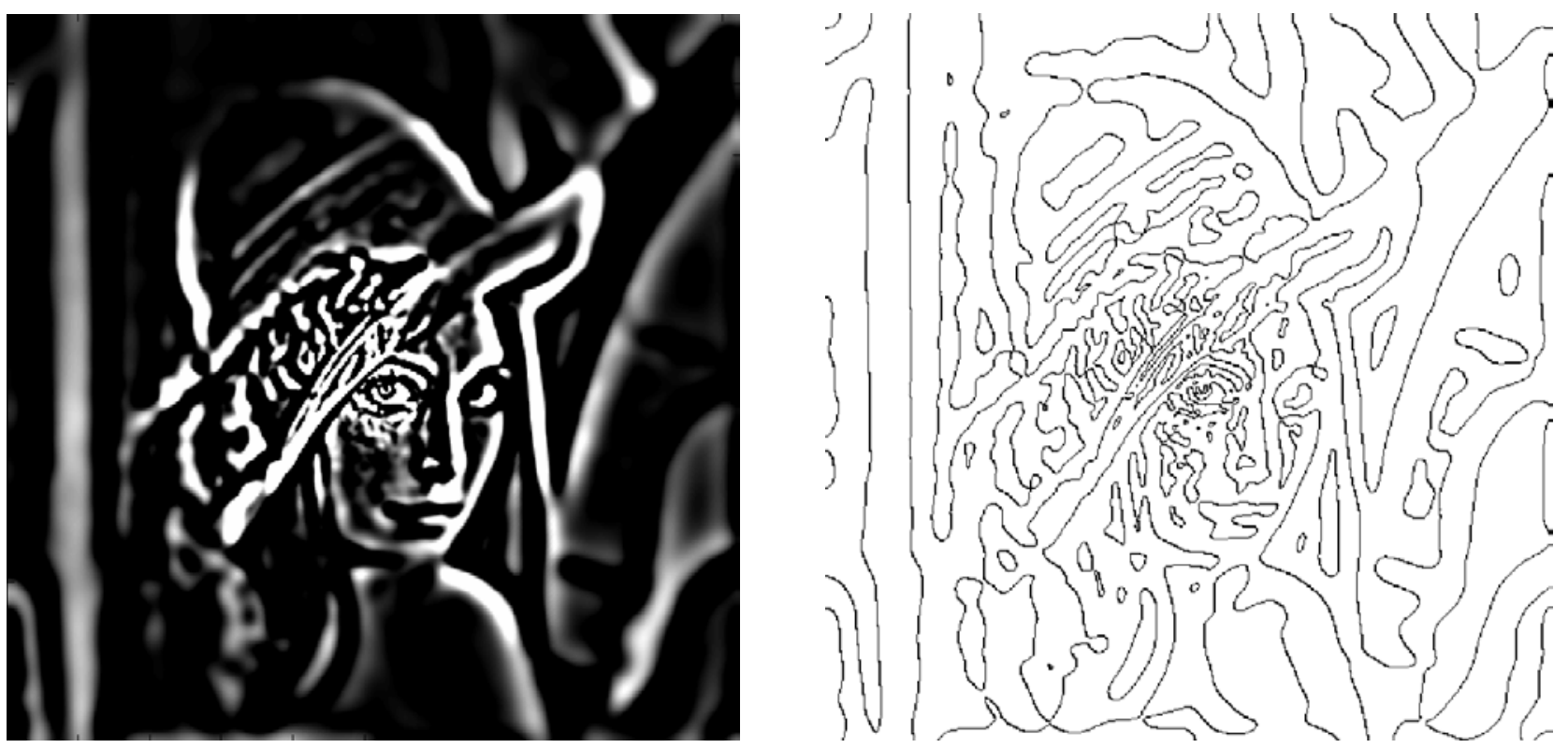

Fig. 11. Left: Scale-variant LoG-filtered Lena image. Right: ZCs computed from Left image.

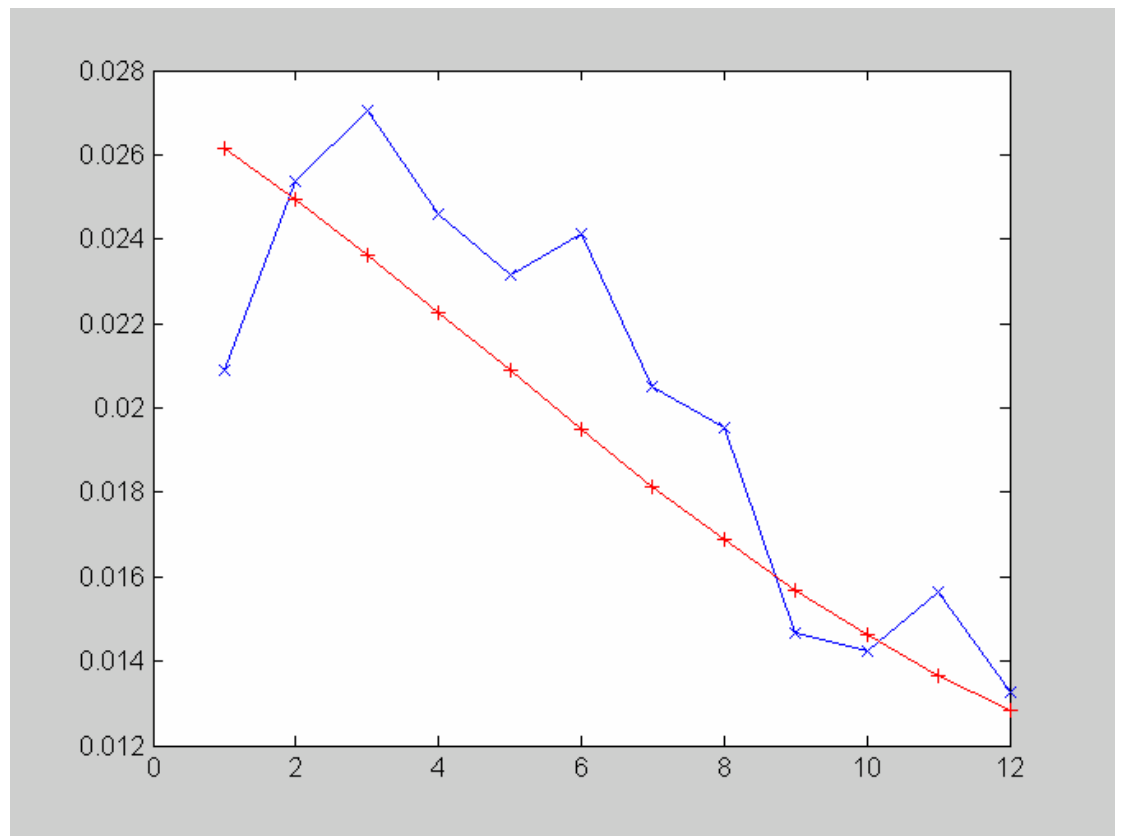

Fig. 12. Plot of theoretical and actual zero-crossing rates averaged over 100 radial directions on image filtered by scale-variant linear Gaussian filter. (Legend: +:Theoretical ZC Rate, $\mathrm{x}$ : Actual ZC Rate) 